\title{
Study on the effect of phototherapy for inhibition of symptoms associated with allergic rhinitis
}

\author{
${ }^{1}$ AgriTech Animal and Plant Research Centre, Warwickshire Colleges, Pershore, Worcestershire, United Kingdom \\ ${ }^{2}$ National Pollen and Aerobiology Research Centre, University of Worcester, Worcester, United Kingdom
}

\section{KEY WORDS}

phototherapy; allergic rhinitis; grass pollen; intranasal; allergy

\section{Corresponding author}

Roy Kennedy

AgriTech research centre

Pershore College, Worcestershire

United Kingdom WR10 3JP

E-mail: rkennedy@warwickshire.ac.uk

Phone: +4403301357212

Doi

10.23822/EurAnnACI.1764-1489.111

\section{Abbreviations}

Total nasal symptom scores, TNSS, allergic rhinitis, AR.

\begin{abstract}
Summary
Previous published work has indicated that treatment of the inside of the nose with certain wavelengths of light can reduce the symptoms of allergic rhinitis. The objective of the study was to compare the efficacy of the phototherapy device on the relief of a range of symptoms provoked by indoor and outdoor allergens. A phototherapy emits visible light ( $m U V / V I S)$ and infrared light, and was compared to a placebo device which did not emit light on two groups of allergic rhinitis sufferers. Rhinophototherapy improved nasal symptoms of allergic rhinitis arising from exposure to indoor and outdoor allergens. The difference in the intensity of symptoms scored at the baseline, and at the final visit for the group using the photoperiod device was significantly lower. The device could potentially help improve the quality of life for allergy sufferers. Phototherapy may be suitable for sufferers either as a replacement therapy or used alongside traditional medication.
\end{abstract}

\section{Introduction}

The nose is the first line of defence against inhaled potentially harmful airborne particles. By acting as a filter, it prevents allergens from reaching the bronchial tree. Allergic rhinitis (AR) results from the inflammation of the nasal lining caused by an allergen, such as pollens, moulds, dust or certain animal danders, which cause symptoms such as nasal irritation, sneezing, rhinorrhoea and nasal blockage (1). These common reactions affect approximately $25 \%$ of the population worldwide and can lead to a reduction in the quality of life, with economic impacts $(2,3)$. AR is often treated using pharmacological products such as antihistamines, corticosteroids or cromolyns either on their own or in a combination depending on the symptoms experienced. However, there are sufferers who do not wish to take medication or for whom medication is contraindicated (4). There are also allergic rhinitis sufferers who wish to reduce the amount of medication that they take, or who find that medication is not sufficient to control their symptoms. One possible method in reducing the dosages of pharmacological products may be to combine their usage with other methods.

Previous published work has indicated that treatment of the inside of the nose with certain wavelengths of light can reduce the symptoms of allergic rhinitis (5). Early studies looked at the effects on perennial / persistent rhinitis and more recent studies $(6,7)$ have looked at the effect on seasonal / intermittent allergic rhinitis. Phototherapy has an immunosuppressive effect and is widely used for the treatment of immune mediated skin diseases. Phototherapy devices are able to inhibit immediate type hypersensitivity reaction in the skin. Intranasal phototherapy is an approach more suitable for treatment of allergic rhinitis. In two open studies, $308 \mathrm{~nm}$ excimer laser and topical PUVA therapy efficiently inhibited clinical symptoms of allergic rhinitis (5). In a randomized, double-blind study combined low dose UVB, 
low dose UVA and visible light proved to be effective in reducing symptom scores for sneezing, rhinorrhea, nasal itching and the total nasal score in ragweed allergic patients. Light wavelength used in phototherapeutic treatment ranged from red light to ultraviolet. Clinical use of intranasal phototherapy appears to be safe and well tolerated. Most studies demonstrated symptomatic improvement in quality of life scores. Treatment with low-energy narrow-band red light phototherapy was demonstrated to improve symptoms in $72 \%$ of the allergic rhinitis patients and the objective improvement was endoscopically demonstrated in $70 \%$ of in comparison with $24 \%$ and $3 \%$, respectively, which was observed in the placebo group (8). These were significantly different. Intranasal phototherapy may represent an alternative treatment of allergic rhinitis and other inflammatory and immune mediated mucosal diseases.

The study reported here investigated the effect of a phototherapy on seasonal / intermittent and perennial / persistent allergic rhinitis symptoms with sufferers who may be affected by one or more allergen sources.

\section{Methods}

\section{Phototherapy test device}

The phototherapy device used in the trial was a Class IIA medical device (Kodec Holdings, Unit D, 20/F., Tai Ping Industrial Centre, Block 1, No 57 Ting Kok Road, Tai Po, New Territories, Hong Kong). The phototherapy device (model Nos mc0018004) has two specific wavelengths which are recommended for reducing the symptoms of Allergic Rhinitis. The device emits visible light (mUV/VIS) and infrared light (660nm8940nm).
The nose probe covers are removed and the on/off button depressed for 1 second, to activate the two wavelengths (figure 1). The two nasal probes are inserted into the nasal cavity by pressing the 2 adjustment buttons. The treatment lasts for 3 minutes and the device automatically turns off once the treatment is completed. The device was used by participants for 3 minutes, twice a day, 5 to 6 hours apart. A placebo device which did not emit light was used on the control group. Participants used the active and placebo device in the morning and evening, although participants were able to fit the use into their normal daily schedules. The study was designed so that participants used the device for 3 weeks with readings taken after 2 weeks (mid study visit-MSV) of use and again after three weeks of use (final study visit -FSV).

\section{Study participant characterisation}

Data and other sample size calculations from previous studies were used to determine the sample size required for this study $(9,10)$. The study comprised of 52 participants with sensitivity to grass and 50 participants with either sensitivity to cat and/ or house dust mite. Participants were provided with a participant information sheet on the nature and scope of the study and were required to submit a signed informed consent form. Inclusions and exclusions were applied. Participants had to be aged 18 years of age or older and sensitive to grass pollen and/or cat dander and/or house dust mite allergen within the previous 2 years. Participants with a history of asthma, nasal deformities / polyposis and sensitive skin were excluded. They were also excluded if they had reported medical conditions or had cold, flu or rhinitis during the initial visit.

Figure 1 - Phototherapy device (Wavelengths 660 nm8 940nm-Model number mc-0018004).

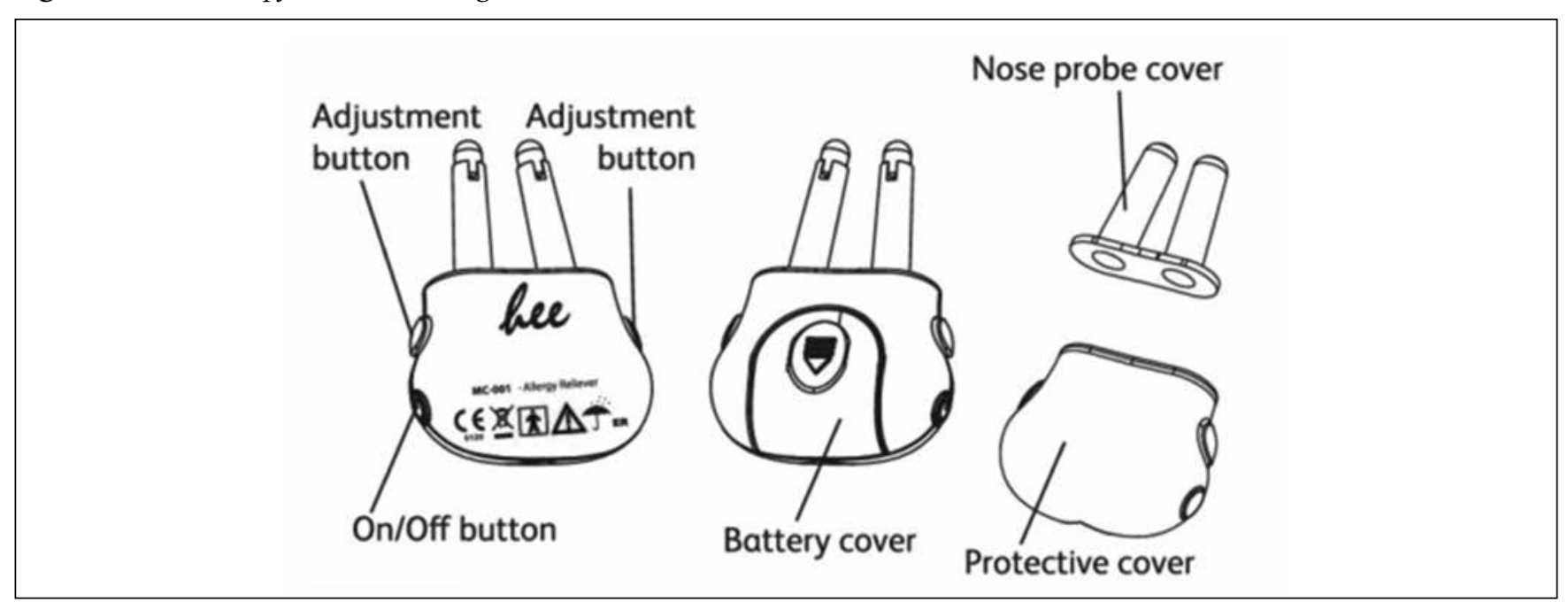




\section{Method used for skin prick testing}

Potential participants were skin prick tested for their sensitivity to grass pollen, cat dander and house dust mite allergen using standard solutions (ALK 7 Abello Soluprick SQ allergen extract 10 HEP) together with a positive control (histamine hydrochloride, ALK Abello Soluprick $10 \mathrm{mg} / \mathrm{ml}$ ) and a negative control (saline solution, ALK Abello Soluprick). The criteria for a positive test was the larger of either a wheal with $3 \mathrm{~mm}$ mean diameter or a wheal with a diameter of $3 \mathrm{~mm}$ greater than the negative control as defined by the World Allergy Organisation (11).

\section{Allergy history}

Participants reported their allergic rhinitis symptom history using scoring scales to ensure they were suitable to participate in the trial (table I) (12). The participant group had 38 people reporting sensitivity to the outdoor allergen (grass pollen) and one or both of the indoor allergens (cat dander and/or house dust mite allergen), 14 people reporting sensitivity to the outdoor allergen (grass pollen) only, and 12 people reporting sensitivity to the indoor allergens (cat dander and/or house dust mite allergen) only. This showed that there were 52 people with allergy to grass pollen, and 50 people with allergy to cat dander and/ or house dust mites (table II). Details of the gender and age breakdown of participants is also shown on table II. At the start of the trial no participant was showing any symptoms associated with allergic rhinitis.

Methods of assessing participant nasal symptoms and participant baseline readings for the trial

As the trial was conducted during the period of the year when grass pollen was not present, participants were not using allergy
Table II - Allergen sensitivity, gender and age of participants in the photoperiod study.

\begin{tabular}{lc}
\hline Allergen & Number in study \\
\hline outdoor (grass) only & 14 \\
\hline indoor (cat/house dust mite) only & 12 \\
\hline $\begin{array}{l}\text { indoor and outdoor (grass and } \\
\text { cat/house dust mite) }\end{array}$ & 38 \\
\hline total in study & 64 (26 males / 38 females) \\
\hline Allergen & Number in study \\
\hline outdoor (grass) & 52 \\
\hline indoor (cat/house dust mite) & 50 \\
\hline Age characteristics of participants & Number \\
\hline 18 - 25 years & 24 \\
\hline 26 - 35 years & 14 \\
\hline 36 - 45 years & 15 \\
\hline 46 - 55 years & 6 \\
\hline 56 - 65 years & 4 \\
\hline $65+$ years \\
(average age 33.7 years)
\end{tabular}

medication. Study participants allergic to cat/house dust mite were asymptomatic at the start of the trial and were not using medication. No trial participants were undergoing immunotherapy. Previously reported methods were used to study nasal symptoms in the trial reported here $(13,14)$. The sum of the Total Nasal Symptom Score (TNSS) is an established method for determining symptom levels of allergic rhinitis. This involves

Table I - Criteria for assessing allergy history of participants.

\begin{tabular}{lll}
\hline Symptom & Score & Criteria \\
\hline scoring of runny nose & $(0-3)$ & nasal blowing $(0-10+$ daily episodes $)$ \\
\hline scoring of itchy nose & $(0-3)$ & rubbing nose $(0-10+$ daily episodes $)$ \\
\hline scoring of blocked nose & $(0-3)$ & nasal stuffiness and mouth breading \\
\hline scoring of sneezing & $(0-3)$ & sneezing $(0-10+$ daily episodes) \\
\hline itchy eyes & $(0-3)$ & rubbing eyes $(0-10+$ daily episodes $)$ \\
\hline watery eyes & $(0-3)$ & watering eyes $(0-10+$ daily episodes $)$ \\
\hline itchy throat & $(0-3)$ & itchy throat (no itching to very itchy) \\
\hline itchy mouth & $(0-3)$ & itchy mouth (no itching to very itchy) \\
\hline itchy ears & $(0-3)$ & itchy ears (no itching to very itchy) \\
\hline
\end{tabular}


evaluating the intensity of nasal symptoms (runny nose, itchy nose, blocked nose, and sneezing) on a scale from 0 to $3(0=$ no symptom, $1=$ mild, $2=$ moderate, $3=$ severe). The TNSS was obtained from the sum of all 4 individual symptom scores, with a total possible score ranging from 0 (no symptoms) to 12 (maximum symptom intensity). Other symptoms recorded were ocular (itchy eyes, runny eyes) and other allergic symptoms (itchy mouth, itchy throat, itchy ears) using the same scale of intensity as used in the TNSS score.

\section{Method of allergen exposure}

A controlled environment test chamber was used in the studies during exposure to allergens. The chamber was set to a typical summer's day with an ambient temperature of $20{ }^{\circ} \mathrm{C}$ with a humidity of $50 \%$. A self-contained allergen challenge chamber which was used to replicate different conditions was located within the environmental test chamber. Previous studies have established allergen challenge chambers as being suitable for studies using allergens (15-17).

Before entering the chamber, each participant was required to put on protective clothing (laboratory coat, hair net, shoe protectors, gloves) to prevent allergen from escaping from the chamber. A tube containing a pre-weighed amount of Timothy grass (Phleum pratense) pollen grains (supplied by Allergon, Denmark) was fitted to the dispersal mechanism. Timothy grass pollen counts can reach between 150 and 400 pollen grains per cubic metre in the UK during summer. Previous studies with grass pollen established that 150 and 400 pollen grains per cubic metre of air are equivalent to high pollen count days in summer. The number of pollen grains required to replicate these field conditions were approximately 6000 grains. Cat dander and house dust mite allergen used levels to replicate equivalent conditions in a typical household and provoke symptoms (18). This equated to approximately 500 particles of both house dust mite $(25 \mu \mathrm{g} / \mathrm{g}$ Der p1) and cat dander $(14 \mu \mathrm{g} / \mathrm{g}$ Fel d1) within the chamber. After 15 minutes the participants left the allergen challenge chamber.

\section{Randomisation}

A random number generator was used to determine the allocation of groups for treatment or placebo group. Participants over the age of 50 were stratified between the treatment group and placebo group as $60 \%$ of rhinitis patients over the age of 50 have symptoms from a non-allergic cause (19). All participants were blinded to the group they were allocated until the end of the study. The study population was made up of 26 males and 38 females. The details of the sensitivity of the participants to different allergens in the treatment and placebo groups are shown in table III.
Table III - Allergen sensitivity breakdown for the treatment group and placebo group.

\begin{tabular}{lccc}
\hline Allergen & $\begin{array}{c}\text { number in } \\
\text { treatment } \\
\text { group }\end{array}$ & $\begin{array}{c}\text { number } \\
\text { in placebo } \\
\text { group }\end{array}$ & Total \\
\hline outdoor (grass) only & 6 & 8 & 14 \\
\hline $\begin{array}{l}\text { indoor (cat/house dust } \\
\text { mite) only }\end{array}$ & 5 & 7 & 12 \\
\hline $\begin{array}{l}\text { indoor and outdoor (grass } \\
\text { and cat/house dust mite) }\end{array}$ & 19 & 19 & 38 \\
\hline
\end{tabular}

Recording participant symptoms during the study

\section{Mid study visit (MSV)}

At the mid study visit, participants had baseline readings taken and then spent 15 minutes in the chamber as per the protocol for the baseline visit. They then had their symptoms monitored for an hour afterwards using the TNSS scale (14).

\section{Final study visit (FSV)}

At the final visit, participants had baseline readings taken and then spent 15 minutes in the chamber as per the protocol for the baseline visit. They were then had their symptoms monitored for an hour afterwards using the TNSS scale (14).

\section{Statistical analysis}

Mann Whitney-U test was used to determine significance ( $p$ $\leq 0.05)$. All statistical tests were carried out two-tailed at $5 \%$ significance levels.

\section{Results}

\section{Effect of phototherapy on eye and nose allergic reactions}

No serious adverse effects were reported either during or after the study from the participants using the protocol applied. Two participants reported that they had severe rhinorrhoea while using their devices, however both of these participants were in the placebo group. One participant reported a faulty device but this was immediately replaced. No problems with using the devices were reported. No problems with compliance with the protocol were reported.

\section{Participant baseline analysis}

A total of 64 data sets were collected. There was a good relationship between the symptoms reported by the participants in their allergy histories and symptoms provoked in the Allergen Challenge Chamber during the baseline visit. There was no dif- 
ference in allergic reactions between groups irrespective of type of allergen used in the allergen challenge (table IVa).

\section{Total nasal symptom scores (TNSS) at final visit}

The TNSS (runny nose, itchy nose, blocked nose, sneezing) was obtained from the sum of all 4 individual symptom scores, with a total possible score ranging from 0 (no symptoms) to 12 (maximum symptom intensity). The total TNSS for the placebo group at baseline was 237 (table IVb), with an overall mean of $7(S D=2)$. The total TNSS for the treatment group at the first visit at the beginning of the trial was 220 , with an overall mean of $7(S D=2)$. There was no significant difference in the TNSS for the treatment group and the placebo group at the first visit at the beginning of the trial $(\mathrm{p}=0.25014)$. There was no significant difference in the TNSS for the treatment group and the placebo group at the first visit at the beginning of the trial for the different categories of allergen (table $\mathbf{I V b}$ ). The total TNSS for the placebo group at the final visit was 209 , with an overall mean of $7(S D=2)$. The total TNSS for the treatment group at the final visit was 142 (table IVb), with an overall mean of $4(\mathrm{SD}=2)$.

The TNSS showed that there was little change in the intensity of symptoms scored at the baseline and at the final study visit for participants in the placebo group ( $\mathrm{p}=0.09492)$; with only a slight change in numbers at each intensity level. The difference in the intensity of all symptoms scored at the baseline and at the final visit for the group using the photoperiod device was significantly lower $\left(\mathrm{p}=0.00024^{* * *}\right)($ table $\mathbf{I V b})$ with a reduction in the intensity of symptoms (table $\mathbf{V})$. The effect of the photoperiod device was observed mainly in the total nasal
Table $V$-TNSS symptom intensities for the placebo and treatment group at baseline and final visit.

\begin{tabular}{lcccc}
\hline & \multicolumn{2}{c}{$\begin{array}{c}\text { Placebo group } \\
\text { numbers }\end{array}$} & \multicolumn{2}{c}{$\begin{array}{c}\text { Treatment group } \\
\text { numbers }\end{array}$} \\
\hline $\begin{array}{l}\text { TNSS } \\
\text { symptom } \\
\text { intensity }\end{array}$ & $\begin{array}{c}\text { number } \\
\text { at } \\
\text { baseline }\end{array}$ & $\begin{array}{c}\text { number at } \\
\text { final visit }\end{array}$ & $\begin{array}{c}\text { number at } \\
\text { baseline }\end{array}$ & $\begin{array}{c}\text { number } \\
\text { at final } \\
\text { visit }\end{array}$ \\
\hline $\begin{array}{l}\text { very mild } \\
(0-2 \text { points })\end{array}$ & 1 & 1 & 0 & 7 \\
\hline $\begin{array}{l}\text { mild symptoms } \\
\text { (3-5 points) }\end{array}$ & 5 & 8 & 7 & 14 \\
\hline $\begin{array}{l}\text { moderate } \\
\text { symptoms } \\
(6-9 \text { points })\end{array}$ & 21 & 20 & 19 & 11 \\
\hline $\begin{array}{l}\text { severe symptoms } \\
(10-12 \text { points })\end{array}$ & 5 & 3 & 6 & 0 \\
\hline \begin{tabular}{l} 
total participants \\
\hline
\end{tabular} & 32 & 32 & 32 & 32 \\
\hline
\end{tabular}

symptom scores (TNSS). Sensitivity to grass represented the major allergenic response group in the trial.

\section{Nasal symptom scores for each allergen sensitivity group}

The outcomes for the different sensitivity groups followed a similar pattern to the overall study

(table VIa and VIb). There was a consistent decrease in the TNSS scores from the baseline visit to the final visit across the three allergen groups (table VIa). This was not observed in the

Table IV - Comparison of treatment and placebo group for a) participant number and mean nasal symptom score with sensitivity type b) TNSS at baseline and final visit for all sensitivities.

a)

\begin{tabular}{cccccc}
\hline allergen type & $\begin{array}{c}\text { number in placebo } \\
\text { group }\end{array}$ & $\begin{array}{c}\text { number in } \\
\text { treatment group }\end{array}$ & $\begin{array}{c}\text { mean score placebo } \\
\text { group }\end{array}$ & $\begin{array}{c}\text { mean score } \\
\text { treatment group }\end{array}$ & $\begin{array}{c}\text { p value } \\
\text { grass only }\end{array}$ \\
\hline $\begin{array}{c}\text { grass and cat/house } \\
\text { dust mite }\end{array}$ & 18 & 6 & 7 & 7 & 7 \\
\hline cat/house dust mite only & 6 & 21 & 7 & 0.60306 \\
\hline
\end{tabular}

b)

\begin{tabular}{cccccc}
\hline severity scores & $\begin{array}{c}\text { baseline placebo } \\
\text { group }\end{array}$ & $\begin{array}{c}\text { final visit placebo } \\
\text { group }\end{array}$ & $\begin{array}{c}\text { baseline treatment } \\
\text { group }\end{array}$ & $\begin{array}{c}\text { final visit treatment } \\
\text { group }\end{array}$ & $\begin{array}{c}\text { p value } \\
\text { TNSS }\end{array}$ \\
\hline 237 & 209 & 220 & 142 \\
\hline overall mean score & 7 & 7 & 7 & 4 & $0.00024^{* * *}$ \\
\hline
\end{tabular}

*** highly statistically significant 
Table VI - Comparison of mean score and Total TNSS for a) placebo and treatment groups at baseline and final visit with allergen type, b) p values for the TNSS between groups.

a)

\begin{tabular}{|c|c|c|c|c|}
\hline \multicolumn{5}{|c|}{ Placebo group } \\
\hline allergen type (baseline) & mean score & mean score (final visit) & total TNSS score (baseline) & total TNSS score (final visit) \\
\hline grass only & 7 & 6 & 57 & 46 \\
\hline cat/house dust mite & 8 & 7 & 58 & 43 \\
\hline \multicolumn{5}{|c|}{ Treatment group } \\
\hline grass only & 7 & 4 & 40 & 21 \\
\hline grass and cat house dust mite & 8 & 5 & 144 & 99 \\
\hline cat/house dust mite & 7 & 4 & 36 & 22 \\
\hline \multicolumn{5}{|l|}{ b) } \\
\hline \multicolumn{2}{|c|}{ grass and cat/house dust mite } & \multicolumn{2}{|l|}{0.3125} & $0.0093^{* *}$ \\
\hline \multicolumn{2}{|c|}{ cat/house dust mite only } & \multicolumn{2}{|l|}{0.6241} & 0.1443 \\
\hline
\end{tabular}

** statistically significant

placebo group, where the TNSS scores either remained the same or changed by only one score. In the analysis of the treatments only the grass and cat/house dust mite allergen group showed a difference that is statistically different $\left(0.0093^{* *}\right)$ (table VIb). However, a p value of 0.1388 (grass only) and 0.1443 (cat and house dust mite only) was observed between the placebo and treatment group at final visit. Although not significantly different, the $p$ value observed at between the placebo and treatment group at baseline visit were $\mathrm{p}=0.6030$ and $\mathrm{p}=0.6241$, respectively (table VIa).

\section{Other allergic responses}

Analysis of the scores for itchy throat and itchy mouth showed that there was no significant difference between the treatment and placebo groups at the baseline visit for either of these two symptoms. At the final visit symptoms of itchy throat ( $\mathrm{p}=$ $0.105)$ and itchy mouth $(p=0.20408)$ were not significantly reduced by phototherapy (table VII). Analysis of the scores for coughing showed that there was no significant difference between the treatment and placebo groups at the baseline visit ( $p$ $=0.2301$ ). At the final visit there was a reduction in the total coughing scores for the treatment group which was found to be statistically significant $\left(\mathrm{p}=0.00341^{* *}\right)$.

\section{Discussion}

Allergic rhinitis is the most frequent atopic response which affects potentially $25 \%-35 \%$ of the adult population and this shows an upward trend (20-22). Previous studies reported using controlled conditions showed that persistent allergic rhinitis patients benefited from adding phototherapy to the medical treatment, using combined UVA, UVB, and visible lights (mUV/vis) (23). In these studies, nasal obstruction, sneezing, rhinorrea, and nasal itching showed statistically significant improvement after rhinotherapy at both 1 st and 3rd month evaluations for each group, when compared with pretreatment

Table VII - Total symptom scores and significance value for itchy throat ( $p$ value).

\begin{tabular}{cccc}
\hline & $\begin{array}{c}\text { total score at } \\
\text { baseline }\end{array}$ & $\begin{array}{c}\text { total score at } \\
\text { final visit }\end{array}$ & p value \\
\hline placebo group & 66 & 60 & \\
\hline $\begin{array}{c}\text { treatment } \\
\text { group }\end{array}$ & 63 & 32 & 0.105 \\
\hline
\end{tabular}


scores (for each symptoms $\mathrm{p}<0.05$ ). The major goal of the study reported here was to determine if there was an effect of phototherapy on symptoms of allergic rhinitis and other allergic responses. Within the clinical trial, the results showed that rhinophototherapy improved nasal symptoms of allergic rhinitis and other allergic symptoms (coughing), which could potentially also alleviate symptoms. This paper reports on a study which was conducted to assess the ability of a photoperiod device in reducing symptoms associated with allergic rhinitis, which has a high incidence rate amongst the population and has the potential to affect quality of life. Medicines such as steroids and anti-histamines are traditionally prescribed as over the counter medical therapies, but there are many sufferers who do not wish to take medication or for who medication is contraindicated. There are also allergic rhinitis sufferers who wish to reduce the amount of medication that they take, or who find that medication is not sufficient to control their symptoms. In other reported studies, the clinical efficacy of rhinophototherapy (doses of $\mathrm{mUV} / \mathrm{vis}$ light for 2 weeks) was compared to the antihistamine, fexofenadine hydrochloride. Rhinophototherapy was significantly better than fexofenadine hydrochloride treatment, with respect to the reduction of individual symptom scores for rhinorrhea, nasal obstruction and total nasal scores (24). Phototherapy may be suitable for sufferers in those cases either as a replacement therapy or used alongside traditional medication. The results of the study reported here indicate that this phototherapy device is particularly effective for the nasal symptoms of allergic rhinitis which fall into the mild/ moderate range. The nasal symptoms consist of a runny nose, blocked nose, itchy nose and sneezing. Seven participants from

\section{References}

1. Skoner DR. Allergic rhinitis: definition, epidemiology, detection, and pathophysiology. Diagnosis. J Allergy Clin Immunol 2001; 108:S2-8.

2. Bauchau V, Durham SR. Epidemiological characterization of the intermittent and persistent types of allergic rhinitis. Allergy 2005; 60:350-353.

3. Zuberbier T, Lotvall J, Simoens S, et al. Economic burden of inadequate management of allergic diseases in the European Union: a GA(2) LEN review. Allergy 2014; 69 (10):1275-1279.

4. Bousquet J, Van Cauwenberge P, Khaltaev N Aria Workshop Group; World Health Organization. Allergic rhinitis and its impact on asthma. J Allergy Clin Immunol 2001; 108:S147-334.

5. Kemeny L. Koreck, A.Ultraviolet light phototherapy for allergic rhinitis. Journal of Photochemistry and Photobiology B: Biology 2007; 87:58-65.

6. Cingi, C, Cakli H, Yaz A, et al. Phototherapy for allergic rhinitis: a prospective, randomized, single-blind, placebo-controlled study. Ther Adv Respir Dis 2010; 4(4):209-213.

7. Lee H, Park MS, Park H, et al. A Comparative Pilot Study of Symptom Improvement Before and After Phototherapy in Korean the treatment group had no symptoms or markedly reduced symptoms at the end of the study in relation to their TNSS and the six participants from this group who had severe nasal symptoms at the start, had them reduced to moderate or mild at the end of the study. All participants in the treatment group had some reduction in one or more of their nasal symptoms.

The phototherapy device was not shown to be effective for the ocular symptoms, but the effect was statistically significant for coughing. There is an indication that the reduction of nasal symptoms can have a secondary effect of helping to alleviate the symptoms of itchy throat and the need for coughing by reducing excessive mucus production.

This study demonstrates that phototherapy may be an effective method for treating and reducing the effects of symptoms for sufferers of allergic rhinitis particularly those affecting the nose. The device could be used in place of other treatments for some sufferers or as an additional treatment for those who find that traditional medication is not sufficient to control their symptoms or when allergen levels are particularly high (25). In this study, phototherapy was shown to be effective in reducing symptoms attributed to several allergens alone or in combination. This makes it particularly useful in the treatment of allergic rhinitis.

\section{Conflicts of interests}

The authors declare that they have no conflict of interest.

\section{Fundings}

Advantage West Midlands
Patients with Perennial Allergic Rhinitis. Photochemistry and Photobiology. 2013; 89:751-757.

8. Neuman I. Finkelstein Y. Narrow-band red light phototherapy in perennial allergic rhinitis and nasal polyposis. J Allergy Clin Immunol 1997; 78:399-406.

9. Emberlin JC. Lewis RA. Pollen challenge study of a phototherapy device for reducing the symptoms of hay fever Curr Med Res Opin. 2009; 25(7):1635-1644.

10. Wolffsohn, JS. Naroo SA. Bilkhu PS. Kennedy R. Robertson L. Effectiveness of Non-Pharmacological Treatments for Acute Seasonal Allergic Conjunctivitis. Ophthalmology 2013; 121(1) DOI: 10.1016/j.ophtha.2013.08.007.

11. Pawanka, R. Walkter Canonica, G. Holgate ST. and Lockey RF. WHO White Book Allergy 2012; 103-104.

12. Linder A. Symptom scores as measures of the severity of rhinitis. Clinical Allergy: Journal of the British Allergy Society 1988; 18(1):29-37.

13. Downie SR. Andersson M. Rimmer J. et al. Symptoms of persistent allergic rhinitis during a full calendar year in house dust mite-sensitive subjects Allergy 2004; 59:406-414. 
14. Hohlfeld JM. Holland-Letz T. Larbig M. et al. Diagnostic value of outcome measures following allergen exposure in an environmental challenge chamber compared with natural conditions. Clin Exp Allergy 2010; 40:998-1006.

15. Badorrek P. Dick M. Hecker H. et al. Anti-allergic drug testing in an environmental challenge chamber is suitable both in and out of the relevant pollen season. J Allergy Clin Immunol 2011; 106:336341. doi: 10.1016/j.anai.2010.12.018.

16. Krug N. Loedding H. Hohlfeld JM. et al. Validation of an environmental exposure unit for controlled human inhalation studies with grass pollen in patients with seasonal allergic rhinitis. Clin Exp Allergy 2003; 33:1667-1674.

17. Day JH. Briscoe MP. Rafeiro E. Ellis AK. Pettersson E. Akerlund A. Onset of action of intranasal budesonide (Rhinocort Aqua) in seasonal allergic rhinitis studied in a controlled exposure model. J Allergy Clin Immunol 2000; 105:489-494.

18. De Lucca SD. O'Meara TJ. Tovey ER. Exposure to mite and cat allergens on a range of clothing items at home and the transfer of cat allergen in the workplace. J Allergy Clin Immunol 2000; 106:874-879.
19. Pinto JM. Jeswani S. Rhinitis in the geriatric population. J Allergy Clin Immunol. 2010; 13: 10.

20. Oettgen HC. Geha RS. IgE in asthma and atopy: cellular and molecular connections. J Clin Invest 1999; 104:829-835.

21. Kay AB. Allergy and allergic diseases: second of two parts. New England Journal Medicine 2001; 344:109-113.

22. Weber RW. Allergic rhinitis. Primary Care 2008; 35:1-10.

23. Tatar EC. Korkmaz, H. Sürenoğlu UA. Saylam G. Özdek A. Effects of Rhinophototherapy on Quality of Life in Persistant Allergic Rhinitis. Clinical and Experimental Otorhinolaryngology 2013; 6:73-77.

24. Garaczi E. Boros-Gyevi M. Bella Z. Csoma, Z. Kemeny L. Koreck A. Intranasal phototherapy is more effective than fexofenadine hydrochloride in the treatment of seasonal allergic rhinitis: results of a pilot study. Photo Photobiology 2011; 87:474-477.

25. Koreck AI. Csoma Z. Bodai L. et al. Rhinophototherapy: A new therapeutic tool for the management of allergic rhinitis. J Allergy Clin Immunol 2005; 115:541-547. 